\title{
Listening to the Music of Reason: Nicolas Bourbaki and the Phenomenology of the Mathematical Experience
}

\author{
TILL DüPPE
}

\begin{abstract}
Denis Guedj: A good proof? Is that something which connects to meaning? I ask the question because often one opposes formalism to meaning.

Claude Chevalley: If meaning is understood as reflection on an existing reality, then it is right to oppose them. But the meaning of which I speak is ONE meaning. (Guidj 22)
\end{abstract}

Claude Chevalley did not know what to say. A mathematical proof can be a good proof, it can be meaningful, but he had no words to describe this meaning. The only thing he could say, as though he had read of Husserl's epoché, was to separate this meaning from the proof representing existing reality. But beyond this, he merely points to this "one" meaning that only those understand who know how it is to go through a good proof. For others, the meaning of mathematics remains unintelligible and, as it were, mystic.

Jean Dieudonné, like Chevalley a prominent member of the French collective of mathematicians named Bourbaki, used an expression from James Sylvester in order to describe this elevated nature of mathematics. He called it "the music of reason" (Sylvester 613 in Weintraub 101). Dieudonné, like Chevalley, eschewed a sophisticated argument about mathematics in its relation with reality. Instead, he wanted to articulate what for him was appealing of the experience of mathematics. Strikingly, he described this experience as a perceptual experience-music as that which we listen to.

Edmund Husserl, when speaking of the internal time consciousness, referred occasionally to the experience of listening to a melody. The identity of a melody is constituted by what he later called a "passive synthesis"- that is, the perception, rather than apperception or conception, of relations. Husserl certainly did not use this example in order to make a 
statement about mathematics (or about music in general), because he, as opposed to Dieudonné, did hold an explicit philosophy of mathematics (Husserl, Crisis and Logik). But in this philosophy, as in most received philosophy of mathematics, Husserl was concerned with the possibility of epistemic certainty, and furthermore scientific objectivity. The Nicolas Bourbaki group, instead, marks a lower watershed in the history of mathematicians' interest in such epistemological questions about their discipline.

In what follows, I thus put aside Husserl's philosophy of mathematics, and use, nevertheless in his spirit, his description of listening to a melody for a phenomenological account of mathematics as the "music of reason." I take Dieudonné's metaphor literally in order to understand the perceptual, in contrast to both the epistemic and discursive, nature of mathematics. In Husserl's sense of the epoché, we thus suspend and "put into brackets," just as the Bourbaki group did, received questions regarding mathematics as a representation of reality in order to disclose the origin of mathematics in our perceptual and affective life: What is the charm of the mathematical experience? What is the specific elevation that fascinates the mathematician? What puts this peculiar experience apart from the rest of life? What makes someone commit to an endeavor so arcane and aloof? In short, how does it feel to do mathematics?

In the literature commenting on the material conditions of mathematics, scholars have put much emphasis on the striking difference between the practices of writing a mathematical proof and its arcane appearance (Livingston, Foundations and "Cultures"; Rota, "Proof" and Beauty; Hersh, "Mathematics"; also earlier, for example, White, "Reality"). In this literature, it is common to oppose the rigor with which mathematics is presented and the contingency that describe its production. This opposition applies to no other mathematics as markedly as to Nicolas Bourbaki. Using a pseudonym and keeping the list of their members secret, while at the same time avoiding any literary or heuristic supplements in their axiomatic presentation of mathematics the gap between the practice and the appearance of their work is strikingly large (Corry, "Bourbaki" and "Origins"). It is this gap, Hersh writes, that "makes possible the preservation of a myth" (127) of mathematics - the myth of its neutrality, its universality, and certainty that "justify and support the institutions of mathematics" (131). Indeed, Bourbaki has considerably contributed to this myth.

The strategy of what follows differs from this literature. Acknowledging the difference between clean appearance and messy production, there is a specific mathematical sensibility underlying this gap. Rather than debunking the myth of universality and certainty, I attempt to show what kind of experience generates this myth in the first place. How must mathematics be experienced in order to induce and 
maintain the image of its elevation? The comparison of mathematics and music, therefore, goes further than considering the impact of aesthetic criteria such as symmetry on choices in mathematics (e.g. McAllister, Beauty). ${ }^{1}$ Sensibility is understood in a transcendental sense as that which "animates" mathematics (Beseelung). Sylvester's initial comparison was indeed written with a transcendental verve: "May not Music be described as the Mathematic of Sense, Mathematic as the Music of reason? The soul of each the same!" (613). Sylvester compared mathematics and music "not merely as having arithmetic for their common parents but as similar in their habits and affections" (613, emphasis added). At this point of the animation of mathematics through our habitual and affective life, where the feeling and the content of mathematics are not yet separate, the elevation and the myth of mathematics have their origin.

This phenomenological reduction to the affectivity of mathematics is not an exercise for its own sake. It does have consequences for those interested in the epistemic and social nature of mathematics. "Bracketing" these issues at first, they will re-emerge from this description in a new light. Specifically, I will show how the idea of the epistemic authority of a mathematical science becomes suggestive without ever being justified. And I will show that this same authority excludes the possibility to assume social responsibility for the uses of this knowledge. With both themes I remain true to Husserl's late work The Crisis of European Science. Husserl argued that due to the modern notion of mathematical science, knowledge lost its "dignity" in being meaningful, which leads to a crisis regarding the role of science for humanity at large $(119,189)$. Modern science is no longer endowed by a lived epistemic interest, which is to say that modern science forgot to be a concrete human accomplishment-it forgot the "life-world." While Galileo represents for Husserl the primal establishment (Urstiftung) of the split of mathematics from a lived epistemic interest, sweepingly stated, Nicolas Bourbaki can be considered its final establishment (Endstiftung). In Bourbaki, as I will conclude from the comparison of listening to a melody and following a proof, the phenomenological problem of modern mathematical science becomes most apparent. But before going through this comparison the following two sections introduce Nicolas Bourbaki's mathematics in the context of the phenomenology of the mathematical experience.

${ }^{1}$ The common arithmetic structure of mathematics and music is omnipresent in all comparisons ever since ancient conceptions of music (see for example Stumpf, Tonpsychologie). Mathematics is mostly thought of giving structure to music rather than music telling us something about mathematics, which is the approach taken in this essay. 


\section{How it is Being Bourbaki}

In the history of mathematics, to begin with, the name of Nicolas Bourbaki stands for a rupture. The work of this illusive collective divides two cultures of mathematics during the first and second half of the $20^{\text {th }}$ century (Dalmedico, "Conflict"). With respect to the first half, Bourbaki is commonly associated with a formalist position although the Bourbaki program shared little with Hilbert's spirit of improving science, as Corry has shown (Corry, "Bourbaki"). With respect to the second half, Bourbaki's work was soon outmoded and replaced, for example, by category theory-partially developed by Bourbaki members such as Grothendieck and Eilenberg. Yet the group influenced the spirit of post WWII mathematics as no other group, certainly regarding the training of mathematicians. Bourbaki has contributed to the separation and isolation of mathematics in its own departments, to which it has handed down a creed of rigor and nothing but rigor. Using Corry's distinction, Bourbaki had a great impact on the "image" of mathematics, while it was hardly effective regarding its "body."

The use of the pseudonym "Bourbaki" was, rather than a mere curiosity, crucial for this influence. The group was founded by seven young French mathematicians associated with the École Normale Superieur in Paris in 1935. The names of the founding members were kept secret for a long time: Jean Dieudonné, Claude Chevalley, Szolem Mandelbrojt, Rene de Possel, Jean Delsarte, André Weil, and Henri Cartan. One formal rule of the group was that membership expires after passing the age of 50. Later participants were as prominent as Samuel Eilenberg, Laurent Schwartz, Alexander Grothendieck, John Tate, and Serge Lang. The collective could survive until today, even though they play a very marginal role. Some say their program was completed, others say it dissolved because of social tensions and conflicts with the state of the art. $^{2}$

The refusal of authorship, in Aristotelian terms, amounts to the denial of assuming a certain ethos as mathematician. Ethos reflects the discursive

\footnotetext{
${ }^{2}$ There is a developed body of literature commenting on the history of the group. One finds first-hand information from interviews for example with Chevalley (in Guedj, "Bourbaki"), or with Cartan (see "Weil" and "Cartan"). Dieudonné ("Work") presents some accounts of the motivations and social dynamics of the group that yet may only apply to his perspective. Regarding the beginnings of the group in Café Capoulade, see Beaulieu ("Parisian"). Standard reference regarding their place in the history of mathematics goes to Leo Corry ("Bourbaki" and "Origins"), but see also Mashaal (Bourbaki). Alternatively, Aubin ("Immortality") provides a fascinating account of how the notion of "structure" in Bourbaki was "in the air" in France during the 1930's and 1940's. In particular, he associates Bourbaki with the structuralism of Saussure and LeviStrauss, and even with Althusser. He also refers to the so-called "potential literature" of a poetry group called "Oulipo" that wrote Bourbakian poems.
} 
identity of a speaker next to both logos reflecting the cogency and pathos reflecting the tone and affective weight of a speech (Aristotle, 1356 a 24). Insisting on their anonymity, the Bourbaki members experienced intellectual forces before, and free from the evocation of an ethos. Their pathos cannot be described in Heidegger's term of a "disposedness" (Befindlichkeit) as no world was disclosed in which they could have "found" themselves as subjects. Bourbaki is the perception of mathematical reason without evoking a subject that has to "stand" for a certain claim. The use of the pseudonym was thus not accidental but programmatic: Bourbaki is mathematics without a mathematician qua person. Bourbaki, so their vision, let mathematics speak for itself, and animated mathematics by itself. They aimed at living "the internal life of mathematics" (Bourbaki, "Architecture" 230).

The absence of the author has been often addressed in the anthropology of mathematics. Eric Livingston, for example, puts much emphasis on the impassive character of what he calls the "lived work of a proof": "a proof is cultivated so as to realize the material proof as a disengaged version, or account, of that proof's lived work" (Foundations 177). Similarly, Reuben Hersh spoke of a front and a back of mathematics, which relate like kitchen and serving area of a restaurant - clean here, and messy there, "formal precise, ordered, and abstract" in the front, and "fragmented, informal, intuitive, tentative," in the back (127-128). For the same reason, Gian-Carlo Rota, in his (slightly improvised) phenomenology of the mathematical proof (Rota, "Proof"), even calls mathematical practices a form of pretending: "Every mathematical proof is a form of pretending. Nowhere in the sciences does one find as wide a gap as that between the written version of a mathematical result and the discourse that is required in order to understand the same result" (Rota, "Proof" 189). Mathematics for Rota is the masking of the practices that brought it about.

Livingston, Hersh, and Rota all point to what also Husserl fascinated about mathematics: the absence of its experience. Mathematical proofs, so the paradox, are constituted as being independent of the acts of constitution. Illustratively speaking, mathematical objects differ from other objects of consciousness in that they are not given in "adumbration" (Abschattung). Mathematical objects are constituted in a different "mode of givenness" from all other objects of consciousness. They reveal a different historicity in that they represent a rupture of the intentional correlation. Mathematical experience, as Husserl wrote in his Ideas I, "does not function as experience":

There are pure eidetic sciences, such as pure logic, pure mathematics, and the pure theories of time, space, motion, and so forth. Throughout, in every step of their thinking, they are pure of all positings of matters of fact; or, equivalently: in them no 
experience as experience, that is, as a consciousness that seizes upon, or posits actuality, factual existence, can assume the function of grounding. Where experience functions in them, it does not as experience. (16)

Because in mathematics there is no "positing of fact" the mathematical experience does not function as experience. The very character of experience as, say, getting to know and coming closer to something, is suspended. The horizon from which mathematical practices are motivated is not "presentiated" (vergegenwärtigt) in mathematics. Experience functions only in absence. The history of sense that leads to a mathematical result, as Derrida will later emphasize in his reading of Husserl, is the "supplement of its validity" (Derrida, "Husserl's Origin").

Much is at stake, thus. The mathematical experience indeed challenges Husserl's principle of principles: intentionality- that objects of consciousness correlate with acts of consciousness. It affects the transcendental rank of experience, comparable with, for example, "death" in Heidegger, or "the other" in Lévinas. In ordinary life, meaning is constituted as a correlate of acts of consciousness (Bewusstseinsakte), which actually occur; they have a history. Constitution in Husserl refers to a history of sense (Sinngeschichte) rather than to the subordination of the content of experience to categories of cognition. To exhibit the transcendental constitution of something is to exhibit the history of acts that preceded their current state of being conscious. " $[\mathrm{H}]$ istory is from the start nothing other than the vital movement of the coexistence and the interweaving of original formations and sedimentations of meaning," Husserl wrote in a famous appendix to the Crisis (371).

But not so in mathematics. A phenomenology of the mathematical experience is interesting precisely to the extent that this experience is "absent" and cannot be read from its product. Phenomenology and mathematics share both an aspect of the phenomenological reduction; the suspension of posing reality. In both phenomenology and in mathematics reality is put into brackets. Yet, in its limitation to forms, mathematics has no language for describing its experiential dimension, that is, as Husserl had said, its "seat of science in life." Bourbaki, as the following section shows, wittingly deprived themselves of such language in order to purify their mathematical experience.

\section{The Silent Joy of Being Bourbaki}

Besides the refusal of authorship of the collective of mathematicians, what was their actual program? The initial idea was to write a modern textbook in analysis, which later developed in an encyclopedic project that attained a foundational status for the whole of mathematics. In the 
introduction to the Elements of Mathematics, Bourbaki's credo reads as follows: "thus, written in accordance with the axiomatic method and keeping always in view, as it were on the horizon, the possibility of a complete formalization, our series lays claim to perfect rigour" (12). This match of the axiomatic method with complete formalization and with the "claim to perfect rigor" describes the heart of Bourbaki's program. They aimed at a new unification of mathematics, which made them announce nothing less than the "solid foundation for the whole of modern mathematics" (v). The new foundation, favoring set theory as the axiomatic language, was supposed to be given by what they called the "mother-structures" of mathematics: topology, order, and algebra (replacing the traditional classification of analysis, differential calculus, number theory, geometry, etc.). With this notion of structures Bourbaki entered the history of mathematics: "mathematical structures become, properly speaking, the only 'objects' of mathematics" (Bourbaki, "Architecture" 225-226; also see Corry, "Bourbaki”).

Such program was already in the mid-1930s a somewhat naïve endeavor. Both set theory and the related axiomatic approach were haunted by well-known paradoxes. In the 1930s, mathematics was not in a state that provided much hope for future unification, to say the least. Bourbaki, however, hardly worried about challenges associated with Hilbert's formalism, since they did not share his epistemic interest in the sciences. For Hilbert, the axiomatic method was used in order to "deepen the foundations of the individual scientific disciplines" (in Corry, "Origins" 262). He held the basic belief that there is a structural interdependence of a plurality of problems that can be put in terms of basic axioms. Axioms represent essential features of an entire field of research, be they epistemological categories (such as implicit definitions or "atoms of knowledge") or basic ontological properties. For Bourbaki, instead, the axiomatic method was a way to avoid taking position regarding the relevance of mathematics for the sciences. It allowed a topdown approach that proceeds by means of mathematical proofs onlywhich were not central for the axiomatic method in Hilbert. "Axiomatization," Dalmedico comments, "had to be combined with an ideology-the desire to free mathematical theory from dependence on physical necessity" (233). The group was indeed biased against the sciences. Pierre Cartier recalls a conversation with André Weil: "You mentioned that in 1926 you were in Göttingen ... in 1926 something happened in Göttingen.' And Weil asked, 'What did happen in Göttingen?' and I said 'Oh! Quantum mechanics!' And Weil said, 'I don't know what that is"" (Senechal, "Silence" 26).

Bourbaki's bias against the sciences went hand-in-hand with an aversion against philosophical quarrels that haunted mathematics at the time. "Bourbaki did not adopt formalism with full philosophical 
commitment, but rather as a façade to avoid philosophical difficulties," Corry commented (Weintraub, Economics 112). Dieudonné reveals this attitude in the following lines: "[W]e believe in the reality of mathematics, but of course when philosophers attack us with their paradoxes we rush to hide behind formalism: "Mathematics is just a combination of meaningless symbols,' and then we bring out Chapters 1) and 2) on set theory" (Dieudonné, "Work" 145). André Weil revealed the same attitude when arguing that mathematics replaces philosophy (Dalmedico, "Conflict" 236). In their official writings, when addressing for example the "philosophical systems" of "Plato, of Descartes, or of Leibnitz, of arithmetization, or of logistics of the $19^{\text {th }}$ century", Bourbaki claimed modesty:

Our task is a more modest and less extensive one; we shall not undertake to examine the relations of mathematics to reality or to the great categories of thought; we intend to remain within the field of mathematics and we shall look for an answer to the question which we have raised [the unity of mathematics], by analyzing the procedures of mathematics themselves. (“Architecture" 222)

Be it Platonism, logicism, formalism, or intuitionism, these philosophical positions about the nature of mathematics arise only after mathematics has already been "accomplished," and given an epistemic interest. In other words, only because one can practice mathematics without holding philosophical beliefs, the nature of mathematics can be contested. For this reason, also anthropologists of mathematics share a skepticism regarding received philosophy of mathematics:

Whether mathematics concerns a domain of ideal, immutable objects, whether it is based on empirical observation, whether mathematics is reducible to formal logic, or whether mathematical truth depends on conventions of definition and reasoning are, for the anthropologist, propositions that require neither assent nor denial (Livingston, "Cultures" 867).

From the point of view of the experience of mathematics, the question of its philosophical limitations does not pose itself. One can engage wholeheartedly in mathematics without ever taking a position about it. Mathematicians are not spontaneously interested in either science or philosophy. They are alien elements in the history of mathematics, even if many mathematicians engaged in philosophy and science.

This silence regarding philosophy and science corresponds with the refusal of social responsibility of the mathematician, which, as argued elsewhere (Düppe, "Talk"; Düppe \& Weintraub, Equilibrium), was vital for the success of mathematics during the ideologically dense times of the early Cold War: "Why do applications (of mathematics) ever succeed? 
Why is a certain amount of logical reasoning occasionally helpful in practical life? Why have some of the most intricate theories in mathematics become an indispensable tool to the modern physicist, to the engineer, and to the manufacturer of atom bombs? Fortunately for us, the mathematician does not feel called upon to answer such questions." (Bourbaki, "Foundations" 2) Bourbaki's philosophical naivety and social irresponsibility was perhaps the distinguishing feature of their program. There is nothing in the writings of Bourbaki that provides a language to understand the meaning of their work.

The surprising fact is that despite these self-limitations the experience of the "internal life of mathematics" was so intense that all members of Bourbaki were highly committed to the project. All Bourbaki members sacrificed their intellectual life without-at least initially-earning personal notice. Strong personal bonds held the group together, and their meetings have repeatedly been described as very vivacious. Dieudonné recalled that anyone who attends for the first time would "always come out with the impression that it is a gathering of madmen. They could not imagine how these people, shouting - sometimes three or four at the same time-could ever come up with something intelligent. It is perhaps a mystery but everything calms down in the end" ("Work" 141). This brings us back to our initial question: the most vivacious experience of mathematics combined with such sternly rigid work? How come? What made the members commit to their project if not the relevance for science or a philosophical program? How come that the liberation from meaning could strongly intensify their intellectual life? Why should one wholeheartedly engage in a project without the possibility of taking a specific position? Being Bourbaki was to be freed from the meaning of mathematics in philosophy and science, so that below its meaningless structures a new dimension of intellectual sensibility could be liberated. It is this sensibility that interests us in the following section.

\section{Listening to a Melody_Following a Proof}

Taking the expression "the music of reason" literally, it is not "Bourbaki" who wrote the Elements of Mathematics. "Bourbaki" only listened. Reason, which is elsewhere an abstract principle of the faculty of judgment, is that which Bourbaki listens to, which they enjoy, and which makes their work feel "sound" and "binding." Reason, far different from all forms it ever took in the history of Western philosophy, can be experienced as a concrete object of perception. Reason becomes sensible.

Though inconceivable in a Kantian tradition, such is no novelty in phenomenology. Speaking of the "music of reason" is to acknowledge what Husserl called the passive synthesis (Husserl, Analyses). Relations between things, before being conceived, are also perceived. Conditions of 
cognition, in other words, are incorporated in order to function as conditions. One basic mode of the perception of relations is that of affectivity, a form of association (196-198). When thinking of an example of the affective constitution of an object, Husserl might have recalled another student of his teacher Brentano, Carl Stumpf, and his main oeuvre on the psychology of the tone (Stumpf, Tonpsychologie). Husserl occasionally referred to the temporal object "melody." When treating a mathematical proof as a temporal object, like a melody, we can discuss the mathematical sensibility, otherwise a mere mystery, explicitly.

What is it to listen to a melody? How do we come to identify a "melody"?" The identity of a melody in contrast to other objects of perception is not the "coinciding" (Deckung) of a variation of experiences that turn out to be the same thing; a melody is not given in "adumbration" (Abschattung). A melody has no "shadows" but is the extended continuation of a moment. The identity of a melody is itself temporal rather than something that turns out to be the same over time. It is only in time that we can know a melody as "this melody." We cannot point to it. We can only listen to it. ${ }^{4}$

A melody served for Husserl as an example of the "temporal fringe" central to the inner-consciousness of time. He speaks of an "animation of the moment" (Husserl, Internal Time 386) in terms of an extended field of retention and protention: the tone we hear is the tone that follows another without that the preceding tone ever anticipates it. Without actively remembering all preceding tones at all moments, without knowing all variations to come, we still listen to "this melody." We do not hear tone (pause) by tone, as though the tone represented the score. Instead, we hear "tone-by-tone," as though the entire melody were the same as the single tones we hear. There is an affective "animation of the moment," which constitutes the duration of a melody. In one tone all other tones are copresent, yet we follow them as this tone spreads out into the others.

I perceive a measure, a melody. I perceive it step by step, tone by tone. I hear and perceive continuously. Accordingly, there exists an enduring, temporally extended act of perceiving. What do I perceive? The first tone sounds. I hear this tone. But I do not hear

\footnotetext{
${ }^{3}$ Though knowing the phenomenological significance of acoustic experiences such as music from scholars such as Stumpf, Husserl did certainly not think of a general theory of music, let alone the appeal of music, when speaking of melodies. The present comparison thus only applies to melodies in contrast to other elements and forms of music, most prominently analyzed by Adorno (Musik). It is difficult to imagine a valid comparison of listening to music and following a proof for music in general.

${ }^{4}$ Carl Stumpf wrote in this context: „Eine Melodie können wir deshalb auch in Gedanken nicht in schnelleren Tempo durchlaufen, als in welcher wir im Stande sein würden, sie zu singen" (Tonpsychologie 152).
} 
merely its quality in a timeless point. The tone endures and in the course of its duration swells in intensity in this way or that, and so on. And then the second tone follows. I continue to hear, and now I hear it. The consciousness of the preceding tone is not erased, however. I can surely observe, "see," that I still keep my intention directed towards the first tone while the second is "actually sounding", is "actually" being perceived. And so it continues. $(171)^{5}$

Listening to a melody, we can "see" it tone by tone. There is an affective, or, as Husserl also said, a hyletic extension that allows us identifying a melody. We are able to remember and anticipate a melody, "know" it, as it were, only by means of this hyletic field; it is this field that distinguishes one from the other melody. After the last tone has faded away, we can keep in grasp the melody as we walk away, at times, with an earworm. ${ }^{6}$

What is it like, then, to follow a mathematical proof? A mathematical proof, too, takes time. Both, a piece of music and a proof can be known only in duration. Knowing a proof is to have followed it once step by step; it cannot be pointed to or understood at a blow at once. One line follows another like an echo, bringing back other lines and giving rise to the following ones. We do not follow line (pause) by line as though their relation represented the principle according to which they were made. We "see" the proof evolving. Without actively remembering all preceding lines at all moments, we still "follow the proof." The cogency of a proof is that each line impresses and evokes the following line. Understanding a proof is following a proof.

What, then, makes the proof appealing? As in music, it is the awaiting of the moment of closure. When the last tone of a piece fades away, when writing QED at the lower right corner of the blackboard, the entire piece of music and the entire proof is manifest in this last moment. At the end of the proof, we can "see" the entire proof not by actually going through it again, but by having just gone through it. We are still "in the impression of" all steps/tones. After a proof and after a piece of music, there is nothing to add-silence. The last accord and the last lines complete

5 One finds a similar passage in Stumpf: "Gegenstand der Wahrnehmung ist ein Einzelnes, aber nicht in seiner Vereinzelung, sondern in seiner Umgebung, welche als Umgebung nebenbei miterfasst wird ... Beim Erfassen einer Melodie ist dieses Besonders-Wahrnehmen sogar immer und notwendig vorhanden, und zwar wird der Regel nach der augenblicklich gegenwärtige Ton besonders wahrgenommen, während zugleich die jüngstvergangenen im Bewusstsein noch vorhanden sind" (Tonpsychologie $6)$.

${ }^{6}$ One finds further remarks on the emotional aspects of melodies, specifically the difference of the listening to a melody for a first or repeated time, see Stumpf ("Musik" 56-58). 
melody and proof. Mathematicians are indeed the only intellectuals who can walk away having completed something; scientists, instead, after giving answers, take home new problems. What if not this intellectual release of a closed proof should have made Bourbaki believe in the timelessness and eternity of their work? A proof is rigorous as nothing remains to add, for once and for all. Such "final solutions" made Bourbaki feel aloof of other deployments of mind. "Unlike anyone else, Bourbaki actively put forward the view that their conception of mathematics was ... in fact the ultimate stage in the evolution of mathematics" (Corry, "Origins" 253).

However there is a difference between proofs and melodies as melodies are not timeless in the same sense as proofs are. The closure of proofs and of melodies is of a different kind. Though there may be nothing to add after a piece of music fades away, melodies can be listened to again, and their affective tonality would be modified. Melodies can get better or worse by being repeated-we cannot get enough of some melodies, others had been played too many times, and others bring us back to when we heard them the first time. We live with melodies. But proofs? Proofs cannot be repeated at all without stultifying themselves. It is hard to guess if Bourbaki, or any other mathematician, write down their proofs multiple times once they were fully given. Some do, certainly in teaching, but the joy of doing so does not add to their validity. The joy of repeating a proof must be secret. For a proof is conclusive in that it is made Once and For All. This Once and For All, Henri Cartan witnessed, was in fact the initiating moment of the foundation of Bourbaki. "One fine day he (André Weil) said to me: 'Now that's enough: Let's meet with some other people to discuss these questions. Let's finalize the answers, and then we will not have to speak of them again.' Thus was born the Bourbaki group" (Cartan, "Weil" 634). Or, to mention another witness of the same fascination, the daughter of Chevalley said the following about her father's passion: "The way my father worked, it seems that this was what counted most, this production of an object which then became inertdead, really. It was no longer to be altered or transformed. Not that there was any negative connotation to this ... [My father] thought of mathematics as a way to put, objects to death for esthetic reasons." (Senechal, "Silence" 26) Mathematical objects never return. This is what makes them beautiful objects of perceptions rather than useful instruments for tackling problems. The moment when proofs appeal is the same moment they vanish, knowing that they would never return with a different face. Once a theorem has been proven, there is nothing left but to turn to a new theorem. If they cause further disquiet, then they are not actual theorems but mere corollaries. We do not live with proofs.

At this point of the difference between a melody and a proof, mathematics gains its epistemic connotations. In its definite closure, 
mathematics grows flanks and a touch of certainty. The fact that mathematical proofs are made once and for all does not only mean they are dead as taxidermic trophies are dead, but also that they can be assumed. Stultifying of being proven again, and requiring to be forgotten, proofs can be assumed without going through them again. They become instruments, as though factual reality had been posited. The rub of the aesthetics of mathematical proofs is thus that the same affections inherent in proofs nourish the belief in "mathematical science"- the dream of a blackboard science that brings about knowledge only by reasoning without actually going through the many facetted ways of experiencing something-apriori knowledge. The dead objects of mathematics endow intellectual life with an affective hyle that nourishes the belief in apodictic judgments. Also in modern science, following Galileo's dream, there is supposedly nothing to add.

The irony is apparent. Although mathematical proofs are appealing for being detached from science, they also reflect the teleology of intellectual life, the wish to cut short the efforts that are necessary for grasping the empirical intricacies of a problem. The axiomatic method as a method of the sciences stands for this shortcut. ${ }^{7}$ On some occasions also Bourbaki dreamt of this epistemic efficiency of mathematics: "for the research worker who suddenly discovers this structure in the phenomena which he is studying, it is like a sudden modulation which orients at one stroke in an unexpected direction the intuitive course of this thought, and which illumines with a new light the mathematical landscape in which he is moving about." (Bourbaki, "Architecture" 227) Bourbaki's mathematics, to stay in this image, flattens the horizon of the world. Distances vanishlike in topology. One can leap from here to there without the effort of actually going through, without actually getting to know. The world itself appears like a "primitive," indifferent like elements. The world becomes a container of things - as in scientific realism as the modern dogma of the scientific profession.

The crux of the mathematical experience is that precisely in its aloofness over science, there is a suggestive force to conflate the value of mathematical rigor and scientific truth. Mathematics becomes the placeholder for the dream of modern science to complete its knowledge. One of the Bourbaki promoters at the University of Chicago, Marshall Stone, was most explicit in this conflation: "science is reasoning;

\footnotetext{
${ }^{7}$ Michel Serres, who worked in his young years on the difference of Bourbaki and classical mathematics, characterized mathematical proofs in terms of "speed" that he inherited in his philosophy when leaping over from poetry to science and back again: "Speed is the elegance of thought, which mocks stupidity, heavy and slow ... mathematics teaches rapid thought ... When you reproach me with 'structure isn't enough; you've got to add all the intermediate steps,' this is not mathematical thought" (Serres, Conversations 67-68).
} 
reasoning is mathematics; and, therefore, science is mathematics" (Stone, "Mathematics" 61). If scientific practices are motivated by the mathematical experience of bringing objects to death, the lived epistemic interest of getting something to know can only be forgotten. Then, scientific practices are guided by that which in fact makes an end to all epistemic interest in the world: apodictic judgments. Then, in both science and mathematics, the passivity of being forced to comply, rather than the engagement of wanting to know, informs our intellectual life.

The question that emerges from the preceding remarks is thus: How different do the urge of evidence and the urge of a proof feel? Affectively, there is only a shadow of a difference between being forced by a full proof or by scientific evidence. As a correlate of discursive practice, obviously, scientific evidence is never complete as proofs are full. After Galileo showed the clergymen the telescope, clamor followed, not silence. What gives gravity to science is not of the same sort as what gives gravity to a proof. A conclusion in science is not a moment of exhalation, but a moment of stammering: What Does This Mean? Yet the notion of "mathematical science" makes the scientist forget this basic discursive character of their intellectual life; it makes scientists unlearn to gain rewards from the contestability of their knowledge. In other words, mathematics deceives the scientist about what makes knowledge interesting. This deception is what Husserl had called the forgetfulness of the life-world. "[Modern science] lacks precisely the knowledge of what is most fundamental, namely, the knowledge of what could procure meaning and validity for the theoretical constructs of objective knowledge and [which] thus first gives them the dignity of a knowledge." (Husserl, Crisis 119)

\section{Conclusion}

The preceding analysis of the affective nature of mathematics sheds a new light on both its epistemic and discursive aspects. Regarding epistemic aspects, we noted a "secret joy": Mathematics draws its appeal from the liberation from the need to assign meaning to it. Below its meaninglessness a new sensibility is liberated. This sensibility, however, has to remain a secret, as I argued when considering the repeatability of mathematical proofs. Hence the possibility of conflating the passivity of following a proof with the force of evidence. The idea of a mathematical science is suggested though never justified by the mathematical experience. Mathematics therefore nourishes a particular notion of knowledge that truth is that which is beyond discourse, that which can be proven once and for all.

In phenomenological terms, the mathematical experience occupies an ambiguous place in-between art and science. It gains affective weight only 
in contrast to an epistemic interest, but at the same time it cannot fully free itself from such interest without stultifying its rigor. Mathematics thus has to "hide behind" science without ever taking over the work of actual reasoning. This secrecy is not only a problem for the scientist who seek to apply mathematics, but also for the mathematician insofar as he or she must face a clash between mathematical life and its discursive reality.

Once more, Bourbaki provide the best example of this clash. In the course of the development of their program, specifically after their success in the 1950s, the increasing personal tensions in the group evermore mocked their elevated appearance (see Corry, "Bourbaki"). Even if their work was supposed to be guided by rigor and nothing but rigor, there were actual pragmatic decisions to be made which proofs to include in, and to exclude from, their encyclopedia. Anonymous approval of the proofs was not always possible and some members were more dominant in the final decisions than others. The members also disagreed on questions such as the relationship between mathematics and logic, and even about the meaning of unification of mathematics. As soon as one not only listens to the music of reason but also composes it as an author, personal differences and power matter. Dieudonné presented, unauthorized by the others, the image we know today. He made Bourbaki the study of "structures." And, after their success, rather mundane interests insinuated their arcane feelings: "Little by little we talked of everyone's career; it was complete decadence," Chevalley commented (Guedj, "Bourbaki” 21).

Behind the music of reason, there was the clamor of actual persons. The secrecy that allowed Bourbaki to be bound by the "internal life of mathematics" could not be kept. They could not maintain the feelings that motivated their project. The discursive reality became an obstacle to their program, and vice versa, their program became an obstacle to the needs of their expressive lives. This was most clearly expressed by Chevalley, who, next to his mathematical life, also had a political life in an anarchist group called Ordre nouveau.

Guedj: Politics seem to have been excluded from Bourbaki. How did you live this dichotomy between your political involvement outside, and your almost complete investment in Bourbaki, above all at a time when in Germany the Nazis were beginning to enjoy themselves to their hearts content?

Chevalley: I don't know what to say. It's a mistake. What I wrote in the political arena never satisfied me completely. It was only in Bourbaki that I was truly satisfied in what I wrote. (Guedj, "Bourbaki" 21)

Mathematics, as Husserl worried at the end of his life, can become an obstacle for one's expressive life, making one forget the motives of one's 
intellectual efforts. Moreover, and thus the risk for humanity at large, it fosters social irresponsibility.

\section{Works Cited}

ADORNO, Theodor W. Philosophie der neuen Musik. Frankfurt a.M.: Suhrkamp, 1978.

ARCHIBALD, R.C. "Mathematics and Music." The American Mathematical Monthly 31, no. 1 (1924): 1-25.

ARISTOTLE. The 'Art' of Rhetoric. Translated by J.H. Freese. Cambridge (MA): Harvard University Press, 1926.

AUBIN, David. "The withering immortality of Nicolas Bourbaki: a cultural connector at the confluence of mathematics, structuralism, and the Oulipo in France." Science in Context 10, no. 2 (1997): 297-342.

BEAULIEAU, Liliane. "A Parisian cafe and ten proto-Bourbaki meetings (1903-1935)." The Mathematical Intelligencer 15, no. 1 (1993): 27-35.

BOURBAKI, Nicolas. "Foundations of mathematics for the working mathematician." Journal of Symbolic Logic, 14, no. 1 (1949): 1-8.

-. "The architecture of mathematics." The American Mathematical Monthly 57, no. 4 (1950): 221-232.

—. Elements of mathematics: theory of sets. Reading: Addison-Wesley, 1968 [1939].

CARTAN, Henri. "André Weil: memories of as long friendship.” Notices of the American Mathematical Society 46, no. 6 (1999): 633-636.

—. "Interview with Henri Cartan." Notices of the American Mathematical Society 46, no. 7 (1999): 782-788.

CORRY, Leo. "Nicolas Bourbaki and the concept of mathematical structure." Synthese 92 (1992): 315-348.

-. "The origins of eternal truth in modern mathematics: Hilbert to Bourbaki and beyond." Science in Context 10, no. 2 (1997): 25397. 
DALMEDICO, Amy DAHAN. "An Image Conflict in Mathematics after 1945." Changing Images in Mathematics: From the French Revolution to the New Millenium. Eds. Umberto Bottazzini and Amy Dahan Dalmedico. London: Routledge, 2001.

DERRIDA, Jacques. Edmund Husserl's Origin of Geometry: An Introduction. Translated by John P. Leavey. Lincoln: University of Nebraska Press, 1989.

DIEUDONNÉ, Jean A. "The Work of Nicholas Bourbaki," The American Mathematical Monthly 77, no. 2 (1970): 134-145.

DÜPPE, Till. „Talk Means Trouble - Don't Talk: Angst und Wissen in ökonomischen Diskursen." Wirtschaftswissenschaft als Oikodizee? Diskussionen im Anschluss an Joseph Vogls Gespenst des Kapitals in Hanno Pahl und Jan Sparsam. Wiesbaden: Verlag Springer, 2013. 91-112.

-. and E. Roy Weintraub. Finding Equilibrium: Arrow, Debreu, McKenzie and the Problem of Scientific Credit. Princeton: Princeton University Press, 2014.

GUEDJ, Denis. "Nicolas Bourbaki, collective mathematician: an interview with Claude Chevalley." Mathematical Intelligencer, 7, no. 2 (1985): 18-22.

HERSH, R. "Mathematics has a front and a back." Synthese 88, no. 2 (1991): 127-133.

HUSSERL, Edmund. Philosophie der Arithmetik. Mit ergänzenden Texten (1890-1901). Den Haag: Kluwer, 1970.

- The Crisis of European Science and Transcendental Phenomenology. Translated by David Carr. Evanston: Northwestern University Press, 1970.

—. Formale und transzendental Logik. Versuch einer Kritik der logischen Vernunft. Den Haag: Kluwer, 1974.

- Analyses Concerning Passive and Active Synthesis: Lectures on Transcendental Logic. Translated by Anthony J. Steinbock. Wien: Springer, 2001. 
-. Ideas Pertaining to a Pure Phenomenological Philosophy, First Book: General Introduction to a Pure Phenomenology. Translated by Frederick Kersten. Collected Works, II. Dordrecht: Kluwer, 1982.

-. On the Phenomenology of the Consciousness of Internal Time, Collected Works, IV. Kluwer (1969). Translated by John Barnett Brough. Collected Works. Dordrecht: Kluwer (1991).

LIVINGSTON, Eric. The Ethnomethodological Foundations of Mathematics. London: Routledge, 1986.

—. "Cultures of proving." Social Studies of Science 29, no. 6 (1999): 867888.

MASHAAL, Maurice. Bourbaki: A Secret Society of Mathematicians. Translated by Anna Pierrehumbert. Providence, RI: American Mathematical Society, 2006.

MCALLISTER, James W. Beauty and Revolution in Science. Ithaca: Cornell University Press, 1999.

PANZA, Marco. "Mathematical Proofs." Synthese 135, no. 1/2 (2003): $119-158$.

PESIC, Peter. "Hearing the Irrational: Music and the Development of the Modern Concept of Number." Isis 101, no. 3 (2010): 501-530.

ROTA, Gian-Carlo. "The phenomenology of mathematical proof," Synthese 111, no. 2 (1997a): 183-196.

—. "The phenomenology of mathematical beauty," Synthese 111, no. 2 (1997b): 171-182.

—. "The pernicious influence of mathematics upon philosophy" Synthese 88 (1991): 165-178.

SENECHAL, M. "The Continuing Silence of Bourbaki: An Interview with Pierre Cartier." The Mathematical Intelligencer 1 (1998): 22-28.

SERRES, Michel and Bruno LATOUR. Conversations on Science, Culture, and Time. Ann Arbor: University of Michigan Press, 1995.

STONE, Marshall. "Mathematics and the Future of Science." Bulletin of the American Mathematical Society 63, no. 2 (1957): 61-76. 
STUMPF, Carl. Tonpsychologie. Band I (1883) und II. (1890). Leipzig: Hirzel.

-. "Die pseudo-Aristotelischen Probleme der Musik." Philosophischhistorische Abhandlungen 3 (1896): 4-85.

SYLVESTER, James J. "Algebraical Researches, Containing a Disquisition on Newton's Rule for the Discovery of Imaginary Roots, and an Allied Rule Applicable to a Particular Class of Equations, Together with a Complete Invariantive Determination of the Character of the Roots of the General Equation of the Fifth Degree, etc." Philosophical Transactions of the Royal Society of London, 154 (1964): 579-666.

WEINTRAUB, Roy. How Economics became a Mathematical Science. Durham (NC): Duke University Press, 2002.

WHITE, Leslie. "The Locus of Mathematical Reality: An Anthropological Footnote." Philosophy of Science, 14 (1947): 289-303. 